\title{
Prevalence of Tuberculosis/HIV co-infection in the state of Ceará: a literature review
}

\author{
João Vitor Caetano Goes 1, 2*, Bruno Jaegger Laranjeira 1
}

${ }^{1}$ Universidade Maurício de Nassau, Fortaleza, CE, Brazil.

${ }^{2}$ Post-Graduation Program in Pathology, Federal University of Ceará, Ceará, CE, Brazil.

${ }^{*}$ Corresponding author: João Vitor Caetano Goes. Américo Rocha Lima Street, $n^{\circ} 689$ - Vila Manoel Sátiro. Zip Code: 60713-240 - Ceará, CE, Brazil. Phone: +55 (85) 9 9803-3239. E-mail: vitorc.biomed@gmail.com.

Received on: May 25, 2021. Accepted on: Jun 5, 2021. Available online: Jun 11, 2021.

\begin{abstract}
Based on literature review, the present study aimed to evaluate data on cases of coinfections between tuberculosis and HIV in the period from 2015 to 2018 in the state of Ceará. Between 2015-1018, there were a total of 16,776 new TB cases in the state of Ceará (Figure 1). When we analyzed the first two years (2015 and 2016), there was a minimal difference, at 1\%, this figure increased in the following year (2017), from $24.02 \%$ to $25.75 \%$, leveraging in 2018 , representing a total of $26.28 \%$ of the total cases of the last 4 years, that is, there was no change above $2 \%$ over the years. However, the number of cases increases gradually. Recognizing the epidemiological status of different regions and locations allows the state to recognize and interpret more efficiently the ways of combating the diseases that so devastate humanity and take the sleep of so many scientists and government leaders. Furthermore, the correct assessment of these data allows us to understand the growth of the disease in the region, and thus help in the process of controlling and informing the population.
\end{abstract}

Keywords: HIV; Tuberculosis; Co-infection; Epidemiology.

\section{Introduction}

Being considered a very old disease, Tuberculosis (TB) has an estimated origin of 150 million years, so it is believed that it was responsible for leading more people to death than any other infectious disease [1]. Its etiological agent is Mycobacterium tuberculosis (MTB), known as Koch bacilli (BK), being a mandatory intracellular microorganism, and can remain alive for years in a granuloma and multiply when the individual's defenses are scarce $[2,3]$.

TB is currently considered to be the most prevalent and infectious disease in the world, being responsible for the death of more than 2 million people per year [4]. It is estimated that at least one third of the world's population is infected with MTB, in Brazil, at least 50 million individuals 
have the agent but it remains in a latent state [5].

Within the epidemiologic context, $\mathrm{TB}$ is related to several factors, including: restriction to health services, little treatment adhering, the resistance that some bacilli present before the medication and the immunosuppression state of infected individuals, either by pharmacolates or by diseases that cause this state [6].

Acquired Immunodeficiencie Virus (HIV) completely modified the context of TB infection, for some years, there was a considerable decrease in levels of bacillus infection, however with the increase in HIV cases around the globe, TB has experienced an increasing increase in cases, in addition to increased morbidity and mortality rates [7].

In this context, the present study aimed to evaluate data on cases of coinfections between tuberculosis and HIV in the period from 2015 to 2018 in the state of Ceará.

\section{Methodology}

A literature search was performed by consulting the following databases: DATASUS, Ministry of Health, World Health Organization (WHO), ScienceDirect, MedLine, and Scielo. The keywords used were selected by listing the following combinations: "Epidemiology", "Coinfection", "Tuberculosis", "HIV" and "Ceará".
The inclusion criteria used in this bibliographic search were: full text, written in Portuguese and English. The articles used were case-control studies, retrospective studies, bibliographic reviews and systematic reviews published between the years 2015 to 2018. From the totality of articles found, all those whose theme and objectives were not related were excluded.

\section{Results (Review)}

The data provided by DATASUS is constantly updated, it is always necessary to analyze the data and wait for a future variation, either for more or less. Between 2015-1018, there were a total of 16,776 new TB cases in the state of Ceará (Figure 1). When we analyzed the first two years (2015 and 2016), there was a minimal difference, at $1 \%$, this figure increased in the following year (2017), from $24.02 \%$ to $25.75 \%$, leveraging in 2018, representing a total of $26.28 \%$ of the total cases of the last 4 years, that is, there was no change above $2 \%$ over the years. However, the number of cases increases gradually $[8,9]$.

The values analyzed for HIV diagnosis, whether positive or negative, are based on all annual TB cases, i.e., DATASUS counts total cases and rescans in positive, negative or unrealized conditions. There are still unconfirmed cases, but they will not participate in this analysis.

In 2015, the percentage of people with $\mathrm{Tb}$ tested for HIV increased, 
reaching $64 \%$. being a lower percentage than that found in 2016, this year, it had $73 \%$ of patients tested for HIV. In 2017, the number of HIV tests in $\mathrm{Tb}$ patients reached 76\%, and the year 2018 surpassed all others. presenting a percentage of $79 \%$ [10].

When analyzing all cases in 2015, a total of $4015 \mathrm{tb}$ cases were analyzed, where 342 were tested positive for HIV, and a total of 1356 patients did not undergo the test. In 2016, a total of 4031 cases were reported, where the rate of unperformed tests dropped to just over 200 cases, reaching 1124 patients, the rate of negatives increased in the same proportion and the positive values practically stagnated [10].

The 2017 data is much higher than previous years. A total of 4320 cases of tuberculosis were analyzed.
Over the years, there has been a considerable decrease in the number of UN-performed HIV tests, at least in 2017, only 913 patients did not take the tests, which symbolizes a drop of 443 cases in 2015. Also in 2017, the number of hiv negatives and positives increased, having the values of 2974 and 364 respectively [10].

In 2018, a total of 4410 cases of $\mathrm{Tb}$ were counted, where only 725 patients did not undergo HIV tests. The number of results negatives returns to increase arriving at 3124 and, still with partial results, the number of positive results is higher than those of the previous year, arriving at 357 [11].

Table 1. Relationship of co-infection with Tuberculosis and HIV between 2015 and 2018 in the Ceará state.

- Tuberculose $\mathbf{n}$ TB-HIV

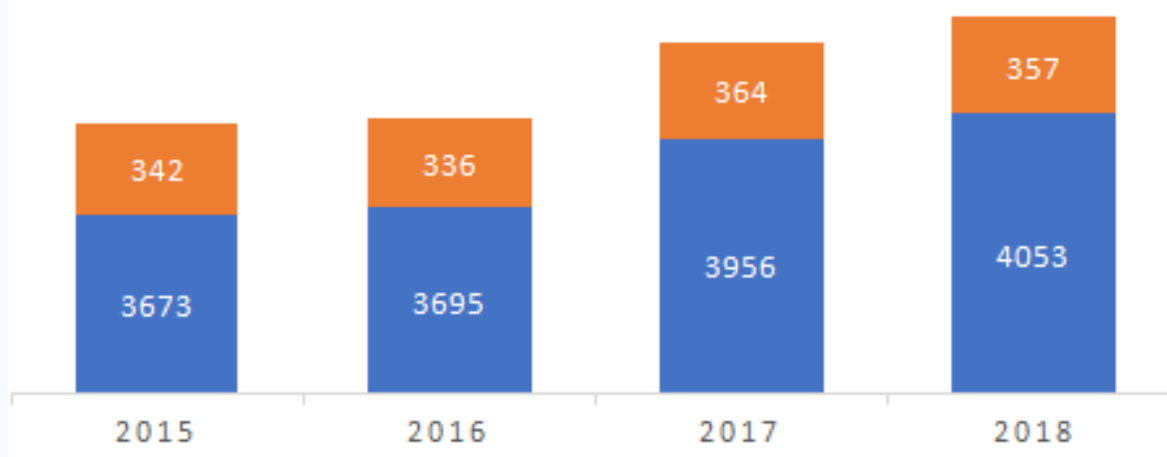

Legend: In the 4 years under analysis, there was an increase in the total number of cases of tuberculosis in Ceará. This growth was accompanied by an increase in the number of new TB-HIV cases. $2015=4015$; $2016=4031 ; 2017=4320 ; 2018=4410$. Source: Adapted from Brazilian Health Department, 2019. 


\section{Discussion and Conclusion}

Over the years 2015 and 2018, there was a significant change in the number of tb/HIV co-infected, the variation for more or less demonstrates an increase in the number of positive results, in addition the increase in the incidence of $\mathrm{Tb}$ in the state can increase these results.

During the 4-year analysis period (2015-2018), there is a noticeable increase in the number of new TB cases, this growth in a first analysis, is directly related to a greater diagnostic efficiency. Another major factor for the considerably growing of the disease was the explosion of new cases of HIV. The immunodepression caused by the virus aggravated the diagnosis control of TB, being currently considered as the main risk factor for tuberculosis.

It is possible to analyze the decay in the number of patients who did not have the hiv diagnosis test, which proves a strengthening of preventive measures, in addition, access to drug treatments has also been recommended within this group of individuals, which facilitates the long-term control of coinfection [8].

Since 1993, tuberculosis has come to be known by the $\mathrm{WHO}$ as a global emergency, since then, many projects have been made with the intuit of complete eradication of the disease. In 2006, the WHO released the Stop TB strategy, which strengthened the DOTS (Directly Observed Treatment Short-
Course) which aims to reduce the prevalence rate of the disease by up to $50 \%$ by 42 in 2015 compared to 1990 [9].

The growth in the number of diagnoses for $\mathrm{Tb}$ facilitates the measurement of co-infection data. The Ministry of Health recommends that all patients who are diagnosed with Tuberculosis undergo rapid HIV testing, the same occurs inversely. People living with HIV/AIDS, because they are 21 to 34 times more likely to contract active $\mathrm{Tb}$, should undergo diagnostic control for tuberculosis [11].

Recognizing the epidemiological status of different regions and locations allows the state to recognize and interpret more efficiently the ways of combating the diseases that so devastate humanity and take the sleep of so many scientists and government leaders. Furthermore, the correct assessment of these data allows us to understand the growth of the disease in the region, and thus help in the process of controlling and informing the population.

\section{References}

[1] Kozakevich G, Maurici R. Tuberculosis: tuberculosis literature review: a review. 2015. 44, 34-47.

[2] Guimarães ABG, Mello DC de, Sousa LÂC de, Silva STF, Souza VF. The history of tuberculosis associated with the socioeconomic profile in Brazil: a review of the literature. CBS. 2018. 3, 43-43. 
[3] Rao M, Ippolito G, Mfinanga S, Ntoumi F, Yeboah-Manu D, Vilaplana C, Zumla A, Maeurer M. Latent TB Infection (LTBI) - Mycobacterium tuberculosis pathogenesis and the dynamics of the granuloma battleground. Int J Infect Dis. 2019 Mar;80S:S58-S61. doi: 10.1016/j.ijid.2019.02.035.

[4] Moutinho ILD. Tuberculosis: immunological aspects in infection and disease. 2010. 21: 42-48.

[5] Busatto C, Reis AJ, Valim ARM, Nunes LS, Carneiro M, Possuelo LG. Active tuberculosis versus Latent tuberculosis: a literature review. Journal of Infection Control. 2015, 4(3):60-64.

[6] Bertolozzi MR, Takahashi RF, Hino P, Litvoc M, France FOS. Control of tuberculosis: a challenge for public health. Rev. Med. (Sao Paulo). 2014. 93:83-89.

[7] Seiscento, M. Tuberculosis in Special Situations: HIV, Diabetes Mellitus and Renal Failure. Lung RJ. 2012. 21: 23-26.

[8] Brazil. Ministry of Health. Department of Health. Tuberculosis Epidemiological Bulletin. Ceará, Ceará. 2018.

[9] Brazil. Ministry of Health. Department of Health. Tuberculosis Epidemiological Bulletin. Ceará, Ceará. 2019.

[10] Brazil. Ministry of Health. Department of Health. HIV/AIDS
Epidemiological Bulletin. Ceará, Ceará. 2018.

[11] Brazil. Ministry of Health. Department of Health. HIV/AIDS Epidemiological Bulletin. Ceará, Ceará. 2019.

[12] Magnabosco, G.T. et al. Tuberculosis control in people living with HIV/AIDS1. Rev. Latino-Am. Nursing. 2016, 24:e2798.

Conflict of interest: The author declares no conflicts of interest.

Acknowledgements: None.

Funding: None.

How to cite this article: Goes JVC, Laranjeira BJ. Prevalence of Tuberculosis/HIV co-infection in the state of Ceará: a literature review. Brazilian Journal of Case Reports. 2021 Abr-Jun;01(2):78-82. 